\title{
Recherches sur des colorations électives d'intérêt parasitologique
}

\section{I. -- Sur deux méthodes de coloration sélective Intérêt dans l'étude des Bilharzioses}

\author{
Par René HOUIN
}

$\mathrm{Si}$, en histologie courante, des colorations telles que l'hémalun-éosine permettent de faire face à toute éventualité, il peut être intéressant, soit dans un but diagnostique, soit dans un but pédagogique, de faire appel à d'autres co.orations, plus sélectives.

C'est pour cette raison qu'ont été étudiées les deux colorations présentées au cours de ce travail. La première fait appel à des propriétés d'acido-alcoo.o-résistance des coques d'œufs d'He'minthes. Elle a été découverte par Brygoo, Capron et Randriamalala en 1959 et a fait l'objet de plusieurs publications de leur part. Seule une légère modification de la technique, d'ailleurs présentée dans un précédent article en collaboration avec J.-J. Rousset et A. Buttner (1962), nous a amenés à la reprendre ici. Cette modification avait été rendue nécessaire par la constation, à l'usage, d'un inconvénient résulttant de l'usage du vert de méthyle : il n'était pas possible, par la technique originelle, de conserver à la fois l'intensité de la coloration et l'intégrité des tissus.

Cette méthode, par coloration à la fuchsine de Ziehl, puis décoloration à l'alcool chlorhydrique, avait permis de mettre en évidence des propriétés d'alcoolo-acido-résistance dans la coque d'un certain nombre d'œufs d'Helminthes. L'intérêt de cette découverte, ne serait-ce qu'au point de vue diagnostique dans les coupes de tissus, était évident. Pour compléter cette technique, les auteurs avaient utilisé le vert de méthyle qui, outre la coloration du fond, permettait une deuxième différenciation, certaines coques d'œufs prenant le vert de méthyle et d'autres pas.

Malheureusement, la teinte obtenue par le vert de méthyle est extrêmement labile sous l'influence de l'alcool et, pour le montage des préparations, il ne restait que le choix entre une lame très décolorée et une lame séchée à l'air. Cette deuxième solution permettait une bonne lecture des coques d'œufs, mais détruisait évidemment toutes les structures histologiques environnantes, réduisant ainsi considérablement l'intérêt de la lame.

La modification proposée consiste simplement en un mordançage par l'acide phosphomolybdique en solution aqueuse à $1 \%$. Cette opération permet au colorant (vert 
ou bleu de méthyle) de résister à l'alcool et ne change en rien les résultats de la méthode. Il devient ainśi facile d'obtenir de très bonnes préparations définitives, bien colorées et respectant l'intégrité des tissus. Il semble en outre que cette coloration soit stable.

Nous reproduisons ici la technique de coloration telle que nous la proposons dans ces conditions :

Après déparaffinage et passage aux alcools :

$1^{\circ}$ Eliminer l'acide picrique des préparations fixées au Bouin dans l'alcool à $70^{\circ}$ saturé de carbonate de lithium pendant cinq minutes environ.

$2^{\circ}$ Laver rapidement.

$3^{\circ}$ Acidifier par l'acide chlorhydrique dilué au dixième pendant une minute.

$4^{\circ}$ Laver.

$5^{\circ}$ Colorer par la fuchsine phéniquée à froid pendant 30 minutes.

$6^{\circ}$ Décolorer jusqu'à teinte rose pâle par l'alcool chlorhydrique à $1 \%$.

$7^{\circ}$ Laver.

$8^{\circ}$ Mordancer à l'acide phosphomolybdique en solution aqueuse à $1 \%$ pendant cinq minutes.

$9^{\circ}$ Colorer sans laver par le bleu de méthyle en solution aqueuse saturée pendant trente secondes à deux minutes selon la fraîcheur de la solution, en surveillant la progression de la coloration.

$10^{\circ}$ Rincer à l'eau.

$11^{\circ}$ Différencier dans l'eau acétifiée à $1 \%$ pendant cinq minutes.

$12^{\circ}$ Rincer dans l'alcool absolu acétifié à $1 \%$.

$13^{\circ}$ Terminer la déshydratation à l'alcool absolu, passer aux bains de toluène et monter au baume du Canada.

Des photographies en couleurs de lames colorées selon cette technique, présentées au cours de la séance, ont permis de constater l'intérêt de cette coloration. Du simple point de vue des Schistosomes, elle permet de reconnaître très facilement, sur coupes histologiques, les œufs des $S$. haematobium de ceux de $S$. mansoni. Les premiers, comme en général ceux qui possèdent un éperon terminal (à l'exception de $S$. nasalis), ont une coque fine et incapable de retenir la coloration par le Ziehl. Les seconds, au contraire, comme tous ceux qui possèdent un éperon latéral, sont franchement acidoalcoolo-résistants. Des résultats comparables ont été obtenus en étudiant des Schistosomes d'Oiseaux.

La seconde coloration se présente comme une coloration d'intérêt beaucoup plus général pour tout ce qui est étude de tissus. Il s'agit en effet d'une coloration trichrome dont les résultats sont très proches de ceux qu'obtient la coloration de Masson, mais qui a l'avantage d'être d'un maniement beaucoup plus simple et beaucoup plus rapide.

C'est en 1950 que Gomori, qui recherchait une façon d'alléger la technique de Masson, découvrit pour la première fois la possibilité d'associer en un seul mélange le 
colorant rouge, le colorant vert et le mordant. Il utilisa à cet effet des colorants différents de ceux qu'utilisait Masson, et qui sont ceux-là mêmes qui font l'objet de cette étude. Cependant, cet auteur continuait à faire au préalable une coloration nucléaire par l'hématoxyline.

En 1951, Wheatley eut l'idée, dans le but de colorer des amibes sur lame, après fixation préalable dans l'alcool polyvinylique, d'utiliser ces colorants sans passage préalable dans l'hématoxyline. Cette technique, appliquée non plus à des amibes mais à des coupes histologiques, s'est révélée très efficace. A notre connaissance, elle n'a pas encore été appliquée en anatomie pathologique parasitaire ou, du moins, elle n'est pas connue en Europe.

Les colorants utilisés sont au nombre de trois :

- le Chromotrope $2 \mathrm{R}$,

- le fast green F C F,

- le light green S F.

Le chromotrope $2 \mathrm{R}$ est un dérivé sulfoné du diazobenzène par copulation avec l'acide chromotropique. C'est un colorant proche du ponceau de xylidine et de l'orange $\mathrm{G}$. Colorant acide, il colore en rouge certains cytoplasmes, en particulier celui des fibres musculaires, certaines sécrétions, et en particulier la cuticule des Helminthes et la coque de leurs œufs. En outre, et quoique acide, il colore la chromatine des noyaux. Son action est favorisée par la fixation des lames au Bouin.

Le fast green et le light green sont dérivés du triphénylméthane. Acides, ce sont des colorants du cytoplasme et ils sont proches du vert de méthyle, du vert lumière et du vert d'iode. Les tissus fibreux leur doivent, sur les coupes, de trancher nettement sur les autres.

Ces trois colorants sont utilisés ensemble, sous forme d'une solution comportant quatre parties de Chromotrope pour une de chacun des autres colorants, et à laquelle sont ajoutés un peu d'acide acétique et un mordant, l'acide phosphotungstique.

On peut, en pratique, préparer la solution de la manière suivante : les constituants secs sont mélangés dans les proportions suivantes :

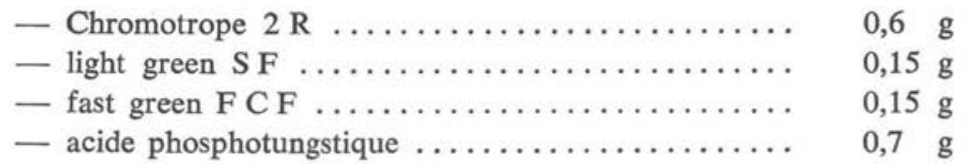

On ajoute alors $1 \mathrm{cc}$. d'acide acétique cristallisable et quelques cc. d'eau distillée. Laisser reposer une demi-heure et compléter l'eau distillée à $100 \mathrm{cc}$. Le colorant obtenu doit présenter une teinte pourpre.

Les lames, préalablement déparaffinées et dépicriquées, sont plongées pendant cinq minutes dans la solution ainsi préparée, puis différenciées quelques secondes dans l'alcool acétique. Cette opération a pour but d'éliminer l'excès du colorant rouge. Nous avions espéré arriver de cette manière à un résultat comparable à celui que nous don- 
nait la première coloration par la fuchsine. Malheureusement il s'est avéré que, dans ce cas, la coque des œufs non-alcoolo-acido-résistants retient le colorant de la même manière que celle des autres œufs. Ce fait peut néanmoins être d'une certaine utilité pour la découverte d'œufs très peu nombreux dans une coupe de tissus.

Cependant, la décoloration prolongée ne se fait qu'exceptionnellement et; dans l'ensemble, c'est à une simple différenciation d'une vingtaine de secondes que l'on fera appel.

La technique de coloration peut donc se résumer ainsi :

Après déparaffinage et passage aux alcools :

$1^{\circ}$ Eliminer l'acide picrique des préparations fixées au Bouin dans l'alcool à $70^{\circ}$ saturé de carbonate de lithium pendant cinq minutes environ.

$2^{\circ}$ Laver rapidement.

$3^{\circ}$ Colorer pendant cinq minutes dans la solution de Wheatley.

$4^{\circ}$ Laver rapidement.

$5^{\circ}$ Différencier dans l'alcool à $90^{\circ}$ acétifié (une goutte d'acide acétique cristallisable pour $10 \mathrm{cc}$. d'alcool) pendant une vingtaine de secondes, en surveillant la coloration de la lame.

$6^{\circ}$ Terminer la déshydratation à l'alcool absolu, passer aux bains de toluène et monter au baume du Canada.

Par conséquent, le temps de coloration proprement dit comporte un bain de colorant de cinq minutes et un bain de différenciation d'une vingtaine de secondes. C'est donc un mode de coloration plus simple et plus rapide que tous les autres.

L'exposé des résultats obtenus par son application à l'étude des altérations tissulaires dues à divers parasites permettra de juger son intérêt.

\section{II. - Application de la coloration de Wheatley \\ à l'étude de quelques processus histo-pathologiques \\ d'origine parasitaire}

\section{Par Alice BUTTNER}

Quelques essais sur coupes histologiques de la coloration trichrome de Wheatley — dont on lira ci-dessus la composition et la technique d'utilisation (R. Houin : « Sur deux méthodes de coloration sélective », ce fasc., p. 137) - nous ont paru suffisamment encourageants pour rechercher plus systématiquement ses modalités éventuelles d'application en pathologie parasitaire. 
Ann. Parasilol. Hum. et Comp.

Tome 39. 1964. No 2

Mémoires R. Houss \& A. Butrwer

1
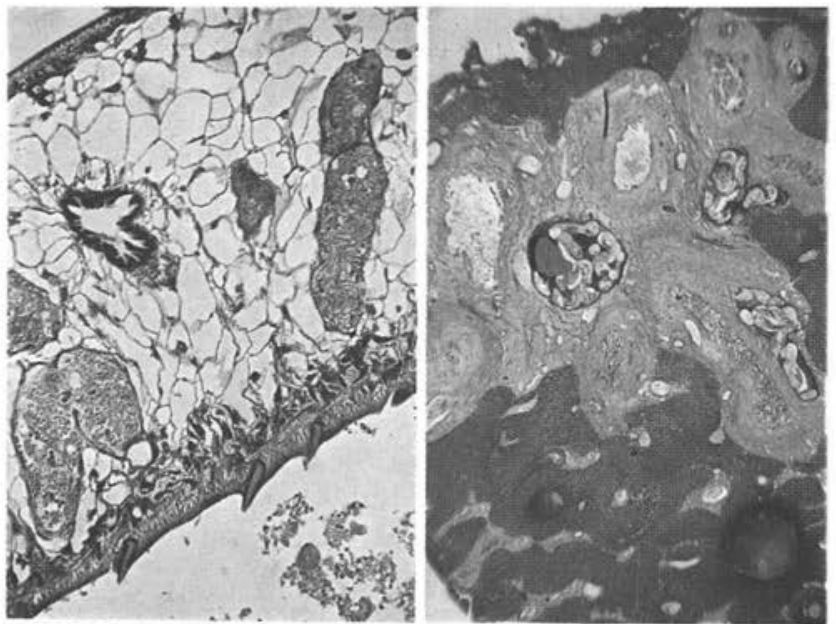

2
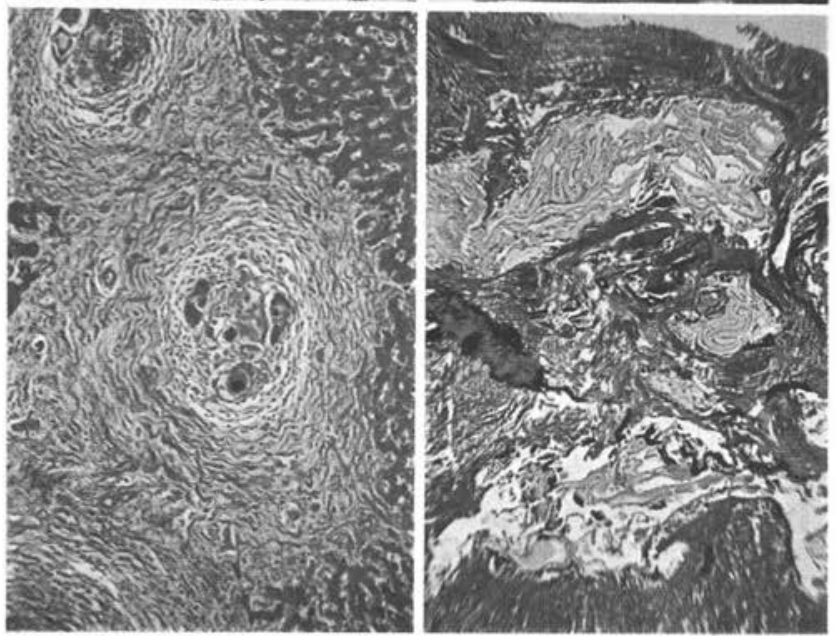

Coloration de Wheatley : 1) coupe de Fasciola hepatica ; 2) coupe de foie de lapin infesté par $S$. japonicum : tubercules fibreux périovulaires ; 3) coupe de foie de lapin à $S$. bovis : fibrose précoce ; 4) coupe de foie de bouf : hydatidose alvéolaire. 



\section{Origine et nature du matériel étudié.}

Le matériel testé a été prélevé sur divers animaux expérimentalement ou spontanément infestés (Souris, Lapin, Hérisson, Bœuf, Mouton, Vison) et dans quelques cas humains.

Les effets de la coloration de Wheatley ont été observés pour des processus histo-pathologiques considérés classiquement comme consécutifs à certaines affections parasitaires: réactions inflammatoire, fibrogène, adénomateuse, d'endothéliite, de dégénérescence graisseuse, de métaplasie, de néoplasie.

L'étiologie des lésions provoquées était soit vermineuse (distomatose, schistosomoses, paragonimose, hydatidoses, capillariose, onchocercose), soit coccidienne (Eimeria stiedai). Dans quelques cas, l'infestation était double (coccidiose et schistosomose ; schistosomose et capillariose), ou unisexuée (schistosomose à vers mâles de $S$. bovis ou $S$. haematobium).

Les organes et tissus qui ont fait l'objet de ces tests de coloration sont le foie, la rate, l'intestin, la vessie, les poumons, la peau.

\section{Résultats.}

Les résultats obtenus varient à la fois avec l'organe considéré et le processus pathologique en cause.

\section{Lésions hépatiques.}

a) FoIEs DOuvés :

Un premier essai a été pratiqué sur des foies de Mouton parasités par Fasciola hepatica. Les résultats sont excellents : l'épais anneau de sclérose qui étreint les canaux biliaires se colore en vert d'autant p'us intense que la sclérose est plus dense, et se détache nettement sur l'architecture lobulaire teintée de violet et de bleu-vert. Les proliférations adénomateuses et les sections du ver qui embolisent les canaux biliaires sont assez finement structurées; en particulier, les cellules réticulées du parenchyme de la douve se colorent en vert, les diverticules cæcaux en violine, tandis que la cuticule, soulignée de bleu, laisse apparaître les épines fortement implantées dans la paroi et teintées de rouge vif (fig. 1).

Un foie douvé humain, infesté de Clonorchis sinensis, a été expérimenté également : les scléroses cholostatiques et la prolifération des canalicules biliaires déterminent des placards de cirrhose qui se colorent en vert d'une intensité croissante avec la progression de la sclérose ; mais les cellules du parenchyme hépatique, souvent en voie de stéatose, prennent alors une teinte mauve, et l'aspect général est moins contrasté que pour les foies d'Ovins ou de Bovins.

Conclusion: L'affinité du tissu conjonctif réactionnel pour les dérivés du triphénylméthane met bien en évidence l'évolution des processus fibrogènes à point de départ biliaire. Par ailleurs, la définiion très satisfaisante des arborescences adénomateuses intra-canaliculaires rend parfaitement compte de leur progression et, par conséquent, du mécanisme de la stase biliaire intra-hépatique. Les deux processus qui concourent fondamentalement à la cirrhose hépatique d'origine distomienne semblent donc clairement démontrés par cette coloration qui remplit ici son rôle didactique. 


\section{b) Foies BILHARZIENS :}

Ils constituent la majeure partie du matériel que nous avons soumis à la coloration de Wheatley.

Foie de Lapin parasité par S. japonicum : l'infestation, à la période d'état, en est au $51^{\circ}$ jour ; elle est objectivée par des amas de tubercules fibreux, disséminés dans le parenchyme, et qui se constituent autour des œufs essaimés dans d'innombrables néocapillaires. La mutation progressive des granulomes péri-ovulaires, parfois centrés par un foyer de nécrose, en fibres régulièrement concentriques, est signalée par le virage de la coloration du violet au vert (fig. 2). La présence de vers adultes - (ils sont relativement peu nombreux) — dans les vaisseaux-porte déterminent des réactions fibreuses périportales qui vont fusionner avec celles issues des tubercules ovulaires, réalisant une importante cirrhose septale et périlobulaire, très significative de l'évolution fibreuse due au japonicum. Les œufs et les vers participent au processus, mais l'influence mécanique et toxique directe des œufs sur le parenchyme hépatique est primordiale et elle est soulignée avec évidence par la coloration de Wheatley.

Foie humain avec $S$. japonicum : infestation sévère et probablement chronique ; quelques vers dans les vaisseaux avec réaction inflammatoire périportale ; mais surtout innombrables amas d'œufs dans le parenchyme, réalisant les vastes travées réactionnelles conjonctives, diffuses entre les lobules, observées expérimentalement dans le cas précédent. Ici encore, l'influence prioritaire de l'action sclérogène des œufs apparaît avec évidence.

Foie de Souris à $S$. mansoni : l'infestation a été faite à partir d'un seul Mollusque, et l'animal a été sacrifié au $50^{\circ}$ jour. Nombreux vers adultes mâles et femelles dans les vaisseaux-porte, prouvant la pluri-infestation du Mollusque utilisé. Faible réaction endothéliale et périportale; pigment diffus dans le parenchyme ; œufs en chapelets ou en amas embolisant les capillaires, sièges de nombreux tubercules fibreux objectivés par la coloration et dont la confluence détermine des plages de sclérose.

Observation doit être faite que l'action sc'érogène des œufs est ici moins intense que pour les œufs de japonicum, et que les vers adultes, dont la participation à l'évolution de la fibrose reste discrète, ont une affinité beaucoup plus grande pour les rameaux porte intra-hépatiques que les vers de japonicum.

Foie de Lapin à $S$. bovis: infestation jeune (21 jours) avec très nombreux vers juvéniles dans les vaisseaux, mais pas encore d'œufs. Une sclérose envahissante se développe autour des rameaux secondaires et tertiaires de la veine porte habités par les vers et encercle les canaux biliaires (fig. 3). Elle forme des nappes conjonctives aux contours arrondis, intensément colorées en vert, et qui fusionnent en progressant dans le parenchyme.

Nous ne nous prononcerons pas sur le déterminisme de cette sclérogénèse évolutive, curieusement précoce. Peut-être s'agit-il d'une sensibilisation du parenchyme hépatique aux produits du métabolisme des jeunes vers en voie de maturation; peut-être d'autres facteurs, d'ordre infectieux ou nutritionnels, sont-ils venus interférer. 
Foie de Hérisson à $S$. bovis : infestation de 3 mois, à la période d'état. Stéatose sévère, intéressant la presque totalité du parenchyme hépatique. Quelques réactions mésenchymateuses et fibroblastiques, objectivées par la coloration, apparaissent au niveau des agrégats d'œufs et des vaisseaux parasités.

Les deux processus semb'ent agir concurremment ; mais, dans ce cas particulier, une stéatose plus ou moins généralisée semb'e s'être développée rapidement et avoir considérablement freiné les processus fibreux d'étiologie vermineuse ; malgré l'abondance des vers adultes dans les vaisseaux, la fibrogénèse périportale est modérée et les nodules péri-ovu!aires demeurent rares. On peut se demander si, lors de la stéatogénèse, il n'y a pas eu une inhibition de la ponte, ou secondairement une fonte des coques ovulaires dans leurs tubercules fibreux.

Foies de Souris à S. haematobium: deux infestations expérimentales unisexuées, à vers mâles, l'une de 3 mois, l'autre de 3 mois et 20 jours, sont responsables de ce parasitisme. On observe essentiellement, dans les deux cas, une stéatose massive; des bulles graisseuses gonflent les cellules et en accroissent le volume; parfois, plusieurs bulles adjacentes se rompent l'une dans l'autre et forment de grandes vacuoles. La coloration permet d'observer la faibie réaction périporta'e en dépit du nombre assez élevé de vers dans les vaisseaux. Même prob.ème que dans le cas précédent en ce qui concerne la chronologie et l'évolution des lésions : la stéatose est-elle imputable à la seule infestation bilharzienne? Dans ce cas, est-elle constante ou transitoire ? Sert-elle de préambule à l'initiation de la fibrose ? Les deux processus sont peut-être antagonistes.

Conclusion: Au point de vue anatomo-pathologique, la coloration de Wheatley met bien en lumière, dans les foies bilharziens, le passage progressif de la première poussée inflammatoire à la sclérose périportale en étoile, puis à la sclérose périlobulaire. Elle donne des endothéliites type «Symmers» et des processus fibrogènes (constitution des fibrilles primaires de réticuline, condensation en ondes concentriques périovulaires, fusion en larges septa jetés entre les espaces-porte) une définition assez nuancée. Mais, surtout, en objectivant par la couleur l'origine périportale de la cirrhose hépatique bilharzienne et la nature de sa progression, qui ne crée aucune perturbation majeure de l'architecture lobulaire, elle justifie l'apparition tardive de la cirrhose atrophique. Celle-ci ne devient effective que lorsque la réunion et l'épaississement des septa conjonctifs réactionnels entraînent mécaniquement une réduction des territoires lobulaires.

\section{c) INFECTIONS MIXTES :}

Les exemples de parasitisme doub'e que nous avons soumis à la coloration sont encore des infestations hépatiques.

Dans un premier cas, il s'agit d'une infestation spontanée de la Souris par Capillaria hepatica sur laquelle a été greffée une infestation expérimentale à $S$. mansoni. Dans les second et troisième cas, il a été procédé à des infestations expérimentales mixtes du Lapin par ingestion de culture d'Eimeria stiedai et par pénétration transcutanée de Cercaires de Bilharzies. 
Premier cas : Foie de Souris avec $C$. hepatica et $S$. mansoni : la coloration met en évidence deux processus pathologiques différents: les co'onies d'œufs de Capillaria, largement essaimés dans le parenchyme hépatique, déterminent une réaction conjonctive qui emprunte le sillage des œufs; amorce de fibrose diffuse non systématisée, sans altération cellulaire importante.

L'infection mansonienne, unisexuée, reste modérée; elle se traduit par une pigmentation abondante, éparse dans le parenchyme, et une réaction périportale qui demeure au stade inflammatoire.

Deuxième cas : Foie de Lapin à Eimeria stiedai et $S$. mansoni : infestations expérimentales de 2 mois, pratiquées simultanément. Dans un foie hémorragique, la coloration met en évidence une importante sclérose primaire et secondaire, d'étiologie biliaire, et une sclérose périportale, plus discrète, noyée dans la cirrhose péricanaliculaire. La sclérose biliaire, d'étiologie coccidienne, est le fruit d'une infestation intense ; la sclérose vasculaire, d'étiologie bilharzienne, est consécutive à la présence dans les rameaux porte de vers à l'exclusion des œufs, l'infestation étant peut-être trop récente, et le Lapin se montrant par ailleurs assez mauvais hôte pour S. mansoni. Les deux processus de fibrose agissent en synergie quoiqu'avec une activité inégale. L'hypervolhémie portale intrahépatique semble être à l'origine de la turgescence variqueuse des petits vaisseaux, parfois de leur rupture et de l'érythrodiapédèse hémorragique observée dans le parenchyme et les canaux biliaires.

Troisième cas : Foie de Lapin parasité par E. stiedai et S. bovis : dans cette observation, l'infestation est plus ancienne (5 mois) que la précédente ; une fibrose évolutive essentiellement bilharzienne se développe autour des œufs et des vaisseaux porte habités par les vers; elle évolue plus modérément autour des canaux biliaires parasités par les Coccidies. Le foie est moins congestionné que dans le cas précédent; nombreux foyers de nécrose dans les tubercules fibreux péri-ovulaires; pas d'érythrodiapédèse hémorragique dans les canaux biliaires.

Conclusion: La coloration de W. met en évidence les points de départ périvasculaire et péricanaliculaire de la fibrogenèse du foie. Observons cependant que dans les trois exemples cités, l'une des infestations prend toujours le pas sur la seconde sans inhiber totalement ses effets pathologiques.

Signalons par ailleurs que, bien qu'il ne s'agisse pas d'un colorant nucléaire, cette technique donne une bonne différenciation des stades schizogoniques et gamétogoniques des Coccidies.

\section{d) FoIes hydatiques :}

Des essais de coloration avec la technique de W. ont été pratiqués sur un foie de Mouton présentant une hydatidose uniloculaire à Echinococcus granulosus et sur un foie de Bæuf avec hydatidose alvéolaire (cas Dévé).

Dans le premier cas (Echinococcose uniloculaire), on obtient une bonne structuration de la membrane réactionnelle (adventice) et des membranes kystiques (anhiste 
et proligère), ainsi que des scolex dans les vésicules proligères (les crochets ne sont cependant pas toujours bien mis en relief). Sur un parenchyme coloré en violet, l'adventice prend assez intensément le vert, tandis que la membrane anhiste stratifiée est plus délicatement différenciée.

Dans le second cas (E. alvéolaire), la nature conjonctive des replis et prolongements racémeux de la membrane anhiste poussés dans le parenchyme hépatique est révélée par la coloration (fig. 4) et rend compte du processus anarchique de la tumeur.

Conclusion: Comme pour les foies douvés et bilharziens, les résultats de la coloration de W. semblent assez révélateurs de l'évolution fibreuse de certaines altérations hépatiques et de la constitution propre des tumeurs hydatiques.

\section{Lésions de la rate.}

Etude des incidences spléniques d'infestations bilharziennes de $S$. bovis chez le Lapin et la Souris. Nous avons procédé à des colorations de coupes de rates des animaux parasités et ne ferons état ici que des résultats obtenus après des infestations relativement anciennes, 3 mois chez le Lapin, 4 mois chez la Souris.

Chez le Lapin, la coloration de la rate fait apparaître de nombreux foyers de nécrose centrés par les œufs, et sièges de processus fibreux circulaires, confluents, qui se substituent peu à peu au tissu lymphoïde et aux corpuscules de Malpighi.

Chez la Souris, même processus, mais moins marqué ; pas de nécrose centro-nodulaire. Malgré la présence des œufs, faibles altérations de la structure de l'organe.

Intérêt de la coloration: il n'existe que lorsque la rate, envahie par les œufs, devient fibreuse.

\section{Lésions intestinales.}

Essai pratiqué sur un intestin de Hamster doré parasité par S. mansoni. Les œufs siègent d'abord dans la sous-muqueuse, plus ou moins noyés dans des tubercules fibreux.

Intérêt de la coloration: elle objective l'éviction des œufs dans la lumière intestinale par les contractions de l'organe, processus qui se traduit par des traînées conjonctives, ponctuées par les œufs et orientées vers la lumière.

\section{Lésions vésicales.}

Cancer bilharzien humain de la vessie. Les résultats ne sont pas probants ; les œufs colorés en rouge sont disséminés dans les tissus ; mais, les réactions fibreuses ne sont pas révélées par la couleur comme dans les cas précédents. Les altérations histologiques et la néoplasie ne sont pas évidentes.

\section{Lésions du poumon.}

Ont été soumis à la coloration de W. un kyste hydatique du poumon (cas humain) et des coupes de poumon de Vison parasité par Paragonimus westermanni. 
Kyste hydatique du poumon. La coloration est ici particulièrement délicate, rendant compte de la structure finement stratifiée de la membrane anhiste, teintée en vert pâle. La membrane réactionnelle fibreuse plus épaisse et plus dense prend davantage le colorant et tranche sur l'architecture alvéolaire du parenchyme pulmonaire.

Poumon de Vison ( $P$. westermanni) : définition très nuancée de la section transversale de la Douve (épines cuticulaires, parenchyme, ventouses) ; mise en évidence des réactions métaplasiques de l'épithélium bronchique et des réactions conjonctives résultant de l'infiltration des œufs dans la paroi, le parenchyme et les alvéoles pulmonaires. La coloration de $\mathrm{W}$. révèle ainsi de façon très satisfaisante l'étiologie des troubles fonctionnels consécutifs à ce parasitisme.

\section{Lésions de la peau.}

Un dernier essai a été effectué sur une biopsie de la peau, au niveau d'un nodule fibreux onchocerquien (Onchocerca volvulus). Résultat discutable. Le nodule fibreux se colore en masse en vert foncé, les filaires en violet sombre. Différenciation assez décevante, nettement surclassée semble-t-il par les colorations classiques.

\section{Conclusion et résumé}

Des différents tests de coloration effectués par la technique de Wheatley, il ressort que :

1) cette méthode est particulièrement révélatrice des processus de fibrose ;

2) parmi les organes ou tissus parasités soumis à cette coloration, c'est dans le foie que les tissus conjonctifs réactionnels apparaissent les mieux contrastés; cependant, résultats satisfaisants également pour l'intestin, la rate, le poumon ;

3) au point de vue histologique, on peut reprocher à la méthode de W. de n'être pas une technique de cytologie fine. Cependant, elle donne une excellente différenciation des structures tissulaires de certains Trématodes (Fasciola, Paragonimus, Schistosoma) et, bien qu'il ne s'agisse pas d'un colorant nucléaire, une définition honorable des stades évolutifs des Coccidies de l'épithélium biliaire ;

4) son mérite essentiel consiste à concrétiser l'histogénèse de certains processus de cirrhose d'étiologie parasitaire (cirrhose biliaire distomienne, cirrhose périportale bilharzienne) et leurs incidences fonctionnelles;

5) cas particuliers : dans des infections hépatiques doubles, cette coloration souligne avec évidence l'activité concurrentielle des deux agents parasitaires sclérogénétiques.

Dans certains cas anciens de bilharziose hépatique expérimentale, elle nous a permis de constater qu'une stéatose massive et persistante semblait inhiber la ponte des vers, ou faire échec à l'action inflammatoire et fibrogénétique des œufs. L'extrême rareté de ces œufs, compte tenu du nombre de vers adultes présents dans les vaisseaux, 
serait en effet insolite dans un parenchyme histologiquement normal, à ce stade de l'infestation.

\section{Bibliographie}

Brooke M.-M., 1961. - Méthode de fixation (A.P.V. fixateur) pour le diagnostic de l'amibiase au laboratoire. Triangle, IV (8), 324-334.

BRYGoo, E. R., 1961. - Substance acido-alcoolo-résistante de la coque de l'œuf de quelques Schistosomes. Arch. Inst. Past. Madagascar, XXIX, 81-82.

Brygoo E.-R., Capron A. et Randriamalala, 1959. - Sur quelques méthodes de coloration sélective des coques d'œufs d'Helminthes parasites de l'homme. Bull. Soc. Path. exot., LII (5), 655-664.

Brygoo E.-R. et Randriamalala J.-Ch., 1959. - Différence de colorabilité au Ziehl entre les œufs de Schistosoma mansoni et ceux de Schistosoma haematibium. Bull. Soc. Path. exot., LII (5), 655-664.

Capron A. et Brygoo E.-R., 1959. - Sur la constitution des œufs d'Helminthes. I. - Présence et formation d'une substance acido-alcoolo-résistante de la coque. Bull. Soc. Path. exot., $L I I$ (1), 26-27.

CAPron A. et Brygoo E.-R., 1960. - Mise en évidence de la formation de la substance acidoalcoolo-résistante de la coque de l'œuf de Schistosoma mansoni. Arch. Inst. Past. Madagascar, XXVIII, 153-154.

Gomori G., 1950. - A rap:d one-step trichrome method. Am. Journ. clin. Path., XX, 661664.

Rousset J.-J., Houin R. et Buttner A., 1962. - Acido-alcoolo-résistance de divers œufs de Schistosomes. Modification de la technique de Brygoo, Capron et Randriamalala. Ann. Parasit. hum. et comp., XXXVII (5-6), 866-869.

WheATley W. B., 1951. - A rapid staining procedure for intestinal amoebae and flagellates. Am. Journ. clin. Path., XXI, 990-991.

Institut de Parasitologie. Faculté de Médecine de Paris Directeur: Professeur L.-Ch. BRUMPT) 